\title{
A narrative review of primary research endpoints of neoadjuvant therapy for lung cancer: past, present and future
}

\author{
Sijia Ren ${ }^{1}$, Anyi Xü ${ }^{2}$, Yilian Lin ${ }^{2}$, D. Ross Camidge ${ }^{3}$, Massimo Di Maio ${ }^{4}$, Raffaele Califano ${ }^{5,6}$, \\ Toyoaki Hida ${ }^{7}$, Antonio Rossi ${ }^{8}$, Nicolas Guibert ${ }^{9}$, Chengchu Zhu $^{1}$, Jianfei Shen ${ }^{1}$ \\ ${ }^{1}$ Taizhou Hospital, Zhejiang University, Taizhou, China; ${ }^{2}$ Taizhou Hospital of Zhejiang Province affiliated to Wenzhou Medical University, \\ Taizhou, China; ${ }^{3}$ Division of Medical Oncology, University of Colorado Anschutz Medical Campus, Aurora, CO, USA; ${ }^{4}$ Department of Oncology, \\ University of Turin/Division of Medical Oncology, Ordine Mauriziano Hospital, Turin, Italy; ${ }^{5}$ Department of Medical Oncology, The Christie \\ NHS Foundation Trust, Manchester, UK; ${ }^{6}$ Division of Cancer Sciences, The University of Manchester, Manchester, UK; ${ }^{7}$ Department of Thoracic \\ Oncology, Aichi Cancer Center Hospital, Aichi, Japan; ${ }^{8}$ Oncology Center of Excellence, Therapeutic Science \& Strategy Unit, IQVIA, Milan, Italy; \\ ${ }^{9}$ Thoracic Oncology Department, Larrey Hospital, University Hospital of Toulouse, Toulouse, France \\ Contributions: (I) Conception and design: S Ren, J Shen; (II) Administrative support: None; (III) Provision of study materials or patients: None; (IV) \\ Collection and assembly of data: All authors; (V) Data analysis and interpretation: All authors; (VI) Manuscript writing: All authors; (VII) Final \\ approval of manuscript: All authors. \\ Correspondence to: Jianfei Shen. Taizhou Hospital, Zhejiang University, Taizhou 317000, China. Email: jianfei051@163.com; \\ Chengchu Zhu. Taizhou Hospital, Zhejiang University, Taizhou 317000, China. Email: zhucc@enzemed.com.
}

\begin{abstract}
Objective: This review summarizes the current status of neoadjuvant therapy and discusses the choice of new clinical research endpoints for non-small cell lung cancer.

Background: Neoadjuvant chemotherapy is a recognized practice in patients with resectable and locally advanced lung cancer. With the introduction of molecular targeted drugs and immune checkpoint inhibitors (ICIs), the overall survival (OS) of patients with lung cancer has been significantly improved, and the original traditional clinical research endpoints are no longer suitable for existing clinical research. In order to accelerate the process of clinical trials and the development and approval of drugs, it is necessary to find suitable alternative indicators as the main indicators of clinical research.

Methods: Therefore, this article focuses on clinical trials using disease-free survival (DFS), progression free survival, and pathological evaluation indicators, pathologic complete response and major pathologic response, as surrogate endpoints. We search related literature through PubMed database and clinical trials through clinicaltrials.gov.

Conclusions: Pathologic complete response and major pathologic response are recommended as surrogate endpoints in the era of neoadjuvant immunotherapy, and secondary endpoints are listed for the prediction of pathological results. In addition, the definitions of major pathological response (MPR) and PCR should be standardized, and a new pathological evaluation standard should be developed, which is applicable to all current treatment methods.
\end{abstract}

Keywords: Neoadjuvant therapy; resectable lung cancer; clinical research endpoint; pathological response

Submitted Jan 14, 2021. Accepted for publication May 28, 2021.

doi: $10.21037 /$ tlcr-21-259

View this article at: https://dx.doi.org/10.21037/tlcr-21-259 


\section{Introduction}

Resectable non-small cell lung cancer (NSCLC) refers to completely resectable stage I, stage II, and part of stage IIIA NSCLC. In recent years, the treatment of resectable NSCLC has made rapid progress, but the 5 -year survival rate remains only $50 \%$, mainly due to distant recurrence. Even completely resected primary tumors less than $1 \mathrm{~cm}$ in size without evidence of nodal spread carry a suboptimal prognosis, with $8 \%$ of patients dying from their disease within 5 years and more than $25 \%$ of patients with stage I recurring within 5 years (1-4). Systemic therapy given before or after surgery (termed neoadjuvant versus adjuvant therapy, respectively) has therefore been actively explored as a means to increase the eradication of micrometastasis in such patients and thereby increase the cure rate.

The option of neoadjuvant therapy is already considered part of routine practice, specifically neoadjuvant chemotherapy or neoadjuvant chemoradiation. However, neoadjuvant molecular targeted therapy, and neoadjuvant immunotherapy remain exploratory. The treatment-related pathological changes of the primary tumor that can be assessed postsurgical resection provide a reliable method for evaluating the impact of neoadjuvant therapy on the tumor. There has already been considerable evaluation of how chemotherapyor chemoradiotherapy-induced pathological responses in the neoadjuvant setting across several tumor types correlate with later outcomes, reflecting their value as surrogate endpoints of clinical benefit. However, because the mechanisms of action of immunotherapy and targeted therapy differ from those of conventional cytotoxic therapies, and the specific histopathological characteristics of response to these different approaches may vary, whether the previous validation of pathological responses as predictors of long-term benefit has required additional investigation. Because neoadjuvant trials offer a means to more quickly assess the effectiveness of drugs many studies of novel agents are evaluated in this setting. Therefore, it is necessary to standardize and unify the research design to explore the research endpoints that better predict the survival benefits of patients after neoadjuvant immunotherapy. This review discusses the endpoints of current clinical trials of neoadjuvant therapy for lung cancer. We present the following article in accordance with the Narrative Review reporting checklist (available at http://dx.doi.org/10.21037/tlcr-21-259).

\section{Method}

To obtain relevant literature, we searched on the PUBMED database and Google website. We search clinical trials through clinicaltrials.gov. The last search date is January 2021. Authors used following combinations of terms: Neoadjuvant therapy, Immune checkpoint inhibitors (ICIs); clinical research endpoint; PFS; DFS; major pathological response (MPR); PCR; positron emission tomography (PET); T-cell response; circulating tumor DNA (ctDNA). Our search was restricted to publications in English language. We retrieved other eligible studies by manual searching of the reference lists of included studies.

\section{Neoadjuvant therapy for lung cancer}

Neoadjuvant therapy has many potential advantages. First of all, neoadjuvant therapy can eliminate all micrometastasis to the greatest extent, thereby increasing the chance of survival. Second, the pathological changes after resection provide a reliable method for evaluating the impact of neoadjuvant therapy on tumors. At least in principle, the detection of the residual tumors provides an opportunity to change (when the response is considered suboptimal) or continue (when the treatment has shown a good activity) the induction program after surgery. Third, the tissues obtained before and after treatment can be used for translational research and utilization of alternative endpoints, thereby significantly speeding up the translation of clinical research results into drug approval (5).

\section{Neoadjuvant chemotherapy}

A meta-analysis of randomized trials of neoadjuvant chemotherapy (5) showed that compared with surgery alone, the overall survival rate (OS) of preoperative chemotherapy was significantly improved [hazard ratio (HR), 0.87; 95\% confidence interval (CI), 0.78-0.96; $\mathrm{P}=0.007]$. This is equivalent to the survival benefit of adjuvant chemotherapy (6). However, after neoadjuvant chemotherapy, 5-year distant recurrence-free survival (DRFS) was found to be $10 \%$; in contrast, adjuvant chemotherapy only provided an absolute improvement of $5 \%$. There is no clear evidence that age, sex, histology (squamous and nonsquamous), and chemotherapy cycle can affect the OS benefit of neoadjuvant chemotherapy.

\section{Neoadjuvant radiotherapy and chemoradiotherapy}

Neoadjuvant radiotherapy alone does not improve resectability or survival. For patients with comorbidities or 
other inoperable reasons, if the patient has stage I NSCLC, stereotactic radiotherapy can be the first choice, with the 5 -year local control rate being about $90 \%$ (7). Neoadjuvant chemoradiotherapy is mainly used for patients with locally advanced (stage III) NSCLC, where the treatment can be definitive or followed by surgery in selected cases, based on tumor response. A clinical trial of stage IIIA (N2) NSCLC patients (8) showed that the median event-free survival (EFS) between a neoadjuvant chemoradiation group (12.8 months, 95\% CI, 9.7-22.9 months) and a neoadjuvant chemotherapy group was similar (11.6 months, 95\% CI, 8.4-15.2 months) $(\mathrm{P}=0.67)$. Another meta-analysis showed that $(9)$, neoadjuvant chemoradiation significantly increased the pathological complete response (pCR) in mediastinal lymph nodes. Neoadjuvant chemoradiotherapy is beneficial to patients with superior sulcus non-small cell lung cancer (SS NSCLC) (10), with the 5 -year OS rate reaching $44 \%$.

\section{Neoadjuvant molecular therapy}

Molecular targeted therapy has greatly improved the treatment of advanced NSCLC. Although a formal demonstration of OS benefit has never been obtained in each single randomized trial versus chemotherapy, Ggefitinib is a first-generation molecular targeted drug directed against epidermal growth factor receptor (EGFR) mutation, which has been shown to double the survival time of NSCLC patients (11). One study evaluated the adjuvant effect of gefitinib combined with chemotherapy in patients with stage II to IIIA NSCLC and EGFR mutation (12). The median disease-free survival (DFS) in the combined treatment group was 28.7 months, while that in the chemotherapy group was 18.0 months (HR, 0.60; 95\% CI, 0.42-0.87; $\mathrm{P}=0.0054)$. This trial and other series have aroused great interest in the use of targeted therapies in neoadjuvant settings. In an open phase II randomized controlled trial (RCT), patients with stage IIIAN2 NSCLC with EGFR exon 19 or 21 mutations were randomly divided into an erlotinib group and a chemotherapy group (13). The ORR for neoadjuvant erlotinib versus GC chemotherapy was $54.1 \%$ versus $34.3 \%$ [odds ratio (OR), 2.26; $95 \% \mathrm{CI}$, 0.87-5.84; $\mathrm{P}=0.092]$. Median progression-free survival (PFS) was also improved (median values 21.5 vs. 11.9 months; $\mathrm{P}=0.003$ ). However, whether targeted therapy is a truly curative modality, such that its benefit persist after the therapy stops remains controversial. Similarly, whether response in the neoadjuvant setting will have the same potential for long-term benefit remains equally unknown.

\section{Neoadjuvant immunotherapy}

ICIs have shown longer OS and better toxicity profile than chemotherapy in advanced NSCLC (14-16). This has inspired the use of ICIs in early resectable NSCLC. Immune checkpoint blockade may be more effective when used as neoadjuvant therapy, because the immune microenvironment of the primary tumor and surrounding lymph nodes is easily recognizable and activated by immune drugs before surgery, thereby increasing $\mathrm{T}$ cell infiltration. At present, a number of clinical trials evaluating programmed cell death protein 1/programmed death-ligand 1 (PD-1/PD-L1) inhibitors as neoadjuvant therapy have been performed. Neoadjuvant nivolumab showed that, of the 20 patients who underwent resection, $9(45 \%)$ achieved major pathological response (MPR) (17). Major pathologic response is defined as a $\leq 10 \%$ portion of surviving tumor cells in different parts of the tumor found during surgery after neoadjuvant therapy. In a phase II clinical trial using atezolizumab, the MPR ratio reached $19.5 \%$ (18), with no increase in the perioperative mortality and postoperative complications showing. The combination of immunotherapy with surgery, chemotherapy, radiotherapy, targeted therapy, and other treatment methods can produce a synergistic effect (19). In a clinical trial of neoadjuvant chemotherapy combined with atezolizumab, 17 of 30 patients (57\%) achieved MPR. with acceptable toxicity and no new or increased surgical complications (20). One meta-analysis evaluating the efficacy and safety of neoadjuvant immunotherapy in resectable NSCLC showed that, compared with neoadjuvant chemotherapy (MPR $<25 \%$, pCR $2-15 \%$ ), the pathological remission rate with neoadjuvant immunotherapy was higher (MPR: OR, 0.59; 95\% CI, 0.36-0.98; pCR: OR, 0.16; 95\% CI, 0.09-0.27).

The incidence of treatment-related adverse events (TRAEs), surgical complications, and surgical delay with neoadjuvant immunotherapy have also proven to be significantly better than those of the neoadjuvant chemotherapy (21). To understand the optimal treatment duration of neoadjuvant immunotherapy and timing of surgery after neoadjuvant treatment, new biomarkers, such as ctDNA may facilitate treatment decision making. In addition, Sepesi et al. (22) reported nodal immune flare (NIF) following neoadjuvant anti-PD-1 and antiCTLA-4 therapy in NSCLC. They defined "the apparent radiographic progression of lymph nodes without pathological evidence of tumor" as NIF and highlighted the importance of judicious restaging of sites of suspected progression after neoadjuvant ICIs prior to definitive 
treatment decisions. Therefore future clinical trials are needed to evaluate further the appropriate schedule for immune checkpoint blockade and to find biomarkers to select patients most likely to benefit from this strategy.

Based on the interesting preliminary results reported above, the results of phase III trials testing the efficacy of neoadjuvant immunotherapy are awaited, in order to better discuss the role of this very promising strategy in clinical practice. As of February 2021, immunotherapy is not currently approved for neoadjuvant use in clinical practice.

\section{The primary endpoints of lung cancer trials}

\section{Features of OS, PFS, and DFS}

In oncology clinical trials, the selection of endpoints needs to consider many factors, such as cancer type, stage, treatment purpose, and expected survival of related diseases. An ideal research endpoint should be clinically relevant, easily measurable, sensitive, specific, low-cost, and reproducible (23). OS is the gold standard for measuring assessing curative effects strategies, and is often used as the primary indicator endpoint of large-scale randomised studies. However, it takes more than 10 years to collect longer follow up and is easily affected by other treatments at progression or patient comorbidities (24). In recent years, in order to accelerate the development of drugs, PFS, DFS, and ORR have become surrogate endpoints for OS.

PFS is one of the most common preferred endpoints in oncology clinical trials. PFS is easy to measure, and compared with death, disease progression occurs earlier and more frequently, which greatly shortens the time period of clinical trials and reduces costs. PFS benefit can predict the benefit of OS, which has been confirmed in colorectal cancer and ovarian cancer. However, surrogacy cannot be extrapolated from one tumor to another, and it should be demonstrated within each specific type of tumor and setting. As a matter of fact, of the phase III RCTs of advanced NSCLC conducted from 1980 to 2010, more trials used PFS as the research endpoint (1980-1990: no progress; 2001-2010 13\%; $\mathrm{P}=0.002$ ) (25).

Similarly to PFS in patients with advanced disease, DFS has been proposed as a surrogate endpoint of OS in patient group undergoing curative treatment, with long-term expected survival. Similar to studies that use PFS, studies using DFS as the end point are less costly and take less time to complete. DFS is most commonly used in the research of adjuvant therapy after radical surgery or radiotherapy.
DFS is currently the main primary endpoint for approval for adjuvant hormone therapy for breast cancer, adjuvant therapy for colon cancer, and adjuvant chemotherapy for breast cancer (23).

Although there is no obvious evidence that surrogate endpoints can predict OS in patients with lung cancer, many meta-analyses have evaluated the correlation between surrogate endpoints and final endpoints, both at the individual and trial level. Fiteni et al. summarized and analyzed current lung cancer trials, which evaluate potential surrogate endpoints for OS (26). They found that in lung cancer trials, the current level of evidence supporting the relationship between OS and potential surrogate endpoints is low. A meta-analysis conducted by Mauguen et al. in surgically resectable and locally advanced NSCLC found that in adjuvant chemotherapy trials, the correlation between individual-level DFS and OS was 0.83 (95\% CI, 0.83), while the correlation between DFS and OS at the test level was 0.92 (95\% CI, 0.88-0.95). In the study of locally advanced disease, they were 0.77 to 0.85 and 0.89 to 0.97 , respectively (27).

\section{pCR as a predictor of $O S$}

Pathologic complete response, which is defined as the absence of any active tumor cells remaining, is a clinical endpoint, especially for neoadjuvant studies. Because pCR can show clinical benefits in a shorter time, especially in high-risk groups, it is especially used to accelerate drug approval. A number of neoadjuvant trials conducted in breast cancer patients have shown that pCR has a strong prognostic correlation with EFS and OS, so pCR has been approved as a surrogate endpoint for breast cancer neoadjuvant treatment studies $(28,29)$. In terms of immunotherapy, it was found that early detected triple-negative breast cancer patients who received pembrolizumab combined with neoadjuvant chemotherapy had a significantly higher pCR than those treated with neoadjuvant chemotherapy (30). Lung cancer trials can learn lessons from studies on other cancers, meaning that pCR can be studied and proposed as a meaningful research endpoint for NSCLC neoadjuvant therapy (Table 1).

More than 20 years ago, Pisters and colleagues published a report after neoadjuvant chemotherapy for patients with stage IIIA N2 NSCLC (31). Of the 73 patients who received preoperative chemotherapy, 9 patients (12\%) achieved pCR. The 3 -year survival rate was $62 \%$, and the 5 -year survival rate was $54 \%$, which is very rare in IIIA N2 
Table 1 Pathologic complete response as a clinical endpoint after neoadjuvant chemotherapy in NSCLC

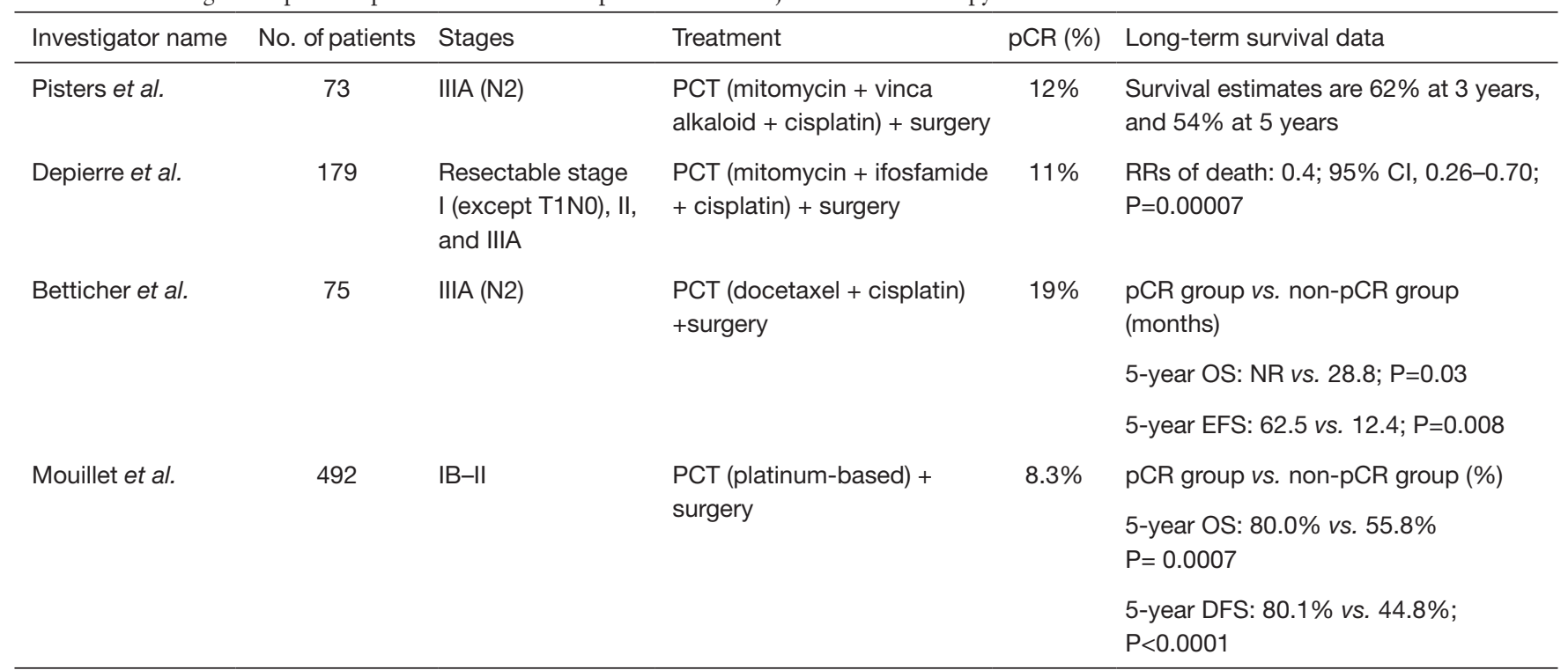

PCT, preoperative chemotherapy; pCR, pathologic complete response; RR, relative risk; OS, overall survival; EFS, event-free survival;

DFS, disease-free survival.

cancer. The report proposed for the first time that $\mathrm{pCR}$ can predict excellent survival and functional levels, and should be regarded as the primary endpoint for evaluating preoperative chemotherapy plans. Depierre et al. examined 179 patients with stage IB-IIIA NSCLC patients who received neoadjuvant chemotherapy (32) and found that the $\mathrm{pCR}$ rate reached $11 \%$, and the relative risk (RR) of death was $0.42(\mathrm{P}<0.001)$. In addition, a multicenter phase II trial in 2003 studied the efficacy of neoadjuvant chemotherapy in locally advanced NSCLC. Of the 90 patients with stage IIIA N2 cancer confirmed by mediastinoscopy, 75 patients underwent surgical resection after receiving neoadjuvant chemotherapy. Complete pathological remission occurred in $19 \%$ of tumor resection patients, and downgrading to N0-1 during surgery could significantly prolong EFS and $\mathrm{OS}(\mathrm{P}=0.0001)$ (33). The subsequent 5 -year followup study indicated that the OS and EFS of 75 patients undergoing surgery were 35 and 15 months, respectively. Local recurrence and distant metastasis occurred in $60 \%$ and $65 \%$ of patients, respectively. The most common sites of distant metastasis were the lungs (24\%) and brain (17\%). The OS rate of the patient is significantly correlated with complete mediastinal lymph node response and clinical and pathological responses (34). There are also many studies that prove that patients who achieve pCR after preoperative chemotherapy can improve the prognosis. However, in these studies, too few pCRs were observed for a full analysis.
Mouillet et al. (35) reported combined analysis of two IFCT randomized trials evaluating neoadjuvant chemotherapy for early NSCLC. The results showed that 41 patients $(8.3 \%)$ achieved pCR and the 5-year OS and DFS rates of pCR patients were $80.0 \%$ and $80.1 \%$, respectively, while those of non-pCR patients were $55.8 \%$ and $44.8 \%$, respectively. This indicated that pCR after neoadjuvant chemotherapy is a strong and favorable prognostic factor for OS (RR, 0.34; 95\% CI, 0.18-0.64) and DFS (RR, 0.29; 95\% CI, $0.16-0.56$ ). However, the incidence of $\mathrm{pCR}$ in cisplatinbased chemotherapy is usually less than $10 \%$, which greatly limits its application as a surrogate endpoint. Therefore, it is necessary to find new pathological evaluation methods.

\section{MPR as a clinical research endpoint}

Major pathologic response is defined as a $\leq 10 \%$ portion of surviving tumor cells in different parts of the tumor found during surgery after neoadjuvant therapy. Many studies have explored MPR is a pathological-related factor suitable for use as a research endpoint in neoadjuvant trials (Table 2).

As early as 2001, Junker et al. conducted a thorough pathological analysis on the resection specimens of 40 locally advanced NSCLC samples that were surgically removed after neoadjuvant chemoradiotherapy. The results showed that for patients with residual tumor tissue $<10 \%$ versus patients with residual tumor tissue $>10 \%$, the median survival time 
Table 2 Major pathologic response as a clinical endpoint after neoadjuvant therapy

\begin{tabular}{|c|c|c|c|c|c|}
\hline Investigator name & $\begin{array}{l}\text { No. of } \\
\text { patients }\end{array}$ & Stages & Treatment & MPR (\%) & $\begin{array}{l}\text { Long-term survival data MPR group vs. } \\
\text { non-MPR group }\end{array}$ \\
\hline Junker et al. & 40 & IIIA and IIIB & $\begin{array}{l}\text { Neoadjuvant chemotherapy (carboplatin, } \\
\text { ifosfamide, etoposide, vindesine) + } \\
\text { radiotherapy ( } 45 \text { Gy) + surgery }\end{array}$ & $67.5 \%$ & $\begin{array}{l}\text { Median survival time: } 36 \text { vs. } 14 \text { months; } \\
\text { 3-year survival rate: } 52 \% \text { vs. } 9 \% \text {; } P=0.02\end{array}$ \\
\hline Pataer et al. & 192 & I-IV & Neoadjuvant chemotherapy + surgery & $19 \%$ & $\begin{array}{l}\text { 5-year OS: } 85 \% \text { vs. } 40 \% \text {; } \mathrm{P}<0.0001 \\
\text { 5-year DFS: } 78 \% \text { vs. } 35 \% \text {; } \mathrm{P}<0.001\end{array}$ \\
\hline
\end{tabular}

MPR, major pathological response; OS, overall survival; DFS, disease-free survival; RFS, recurrence-free survival.

was 36 and 14 months, respectively, while the 3 -year OS was $52 \%$ and $9 \%$, respectively $(\mathrm{P}=0.02)$. Tumor tissue residues of $\leq 10 \%$ caused by treatment are essential to achieve favorable long-term results (36). Later, a retrospective study attempted to determine whether the histopathological remission of NSCLC patients after neoadjuvant chemotherapy was related to OS. The study performed pathological evaluation of tissue specimens from 192 patients with stage I-IV NSCLC after surgical resection (37). The results showed that different tumor cell survival rates and surgical pathological stages were related to OS $(\mathrm{P}=0.005)$ and $\mathrm{DFS}(\mathrm{P}=0.01)$. Compared with patients with surviving tumor cells $>10 \%$, patients with surviving tumors $\leq 10 \%$ had significantly prolonged long-term OS (5-year OS: $40 \%$ vs. $85 \% ; \mathrm{P}<0.0001)$ and DFS (5-year DFS: $35 \%$ vs. $78 \%$; $\mathrm{P}<0.001)$. This suggests that the histopathological evaluation of resected specimens after neoadjuvant chemotherapy can predict survival. They then investigated whether tumor response measured by computed tomography (CT) and evaluated using Response Evaluation Criteria in Solid Tumors (RECIST) correlated OS in patients with NSCLC after neoadjuvant chemotherapy and surgical resection. The results showed that compared with the tumor RECIST remission measured by CT $(\mathrm{P}=0.03)$, tumor pathological remission $(\leq 10 \%$ vs. $>10 \%$ live tumor; $\mathrm{P}=0.002$ ) had a stronger predictive effect on OS (38). In a phase II clinical trial of 50 patients with stage IB-IIIA NSCLC who were treated with neoadjuvant chemotherapy and bevacizumab, patients who achieved MPR during surgery had a better 3-year survival rate than patients who did not achieve MPR ( $100 \%$ vs. 49\%; $\mathrm{P}=0.01)$ (39). After that, an editorial signed by several American oncologists, surgeons, and pathologists suggested that the MPR should be included as a surrogate endpoint for future neoadjuvant trials of resectable lung cancer (40). However, the validity of this in relation to novel therapies, just as with $\mathrm{pCR}$, remains under investigation.

MPR is currently the primary endpoint of many ongoing trials of neoadjuvant immunotherapy. This will help determine the effectiveness of this endpoint as a surrogate for survival. In 2018, Bott et al. published the preliminary results of the histopathological response characteristics and immune-mediated tumor clearance characteristics of 20 patients with NSCLC who received nivolumab neoadjuvant treatment and obtained complete tumor resection and chest lymph node resection specimens (41). The results showed that the MPR reached 45\%, 8 patients (40\%) had a decrease in pathological stage, and 2 patients reached pCR. At 18 months after the operation, the patient's recurrence-free survival (RFS) reached an exciting $73 \%$, and no increase in mortality and disease recurrence rate was observed in the perioperative period. The NEOSTAR trial was a randomized controlled study that compared the efficacy of nivolumab monotherapy and nivolumab combined with ipilimumab dual-immune neoadjuvant therapy in patients with resectable NSCLC (42). Recent reports showed that in the single-drug group (23 patients) and the dual-drug group (21 patients), the MPR rates were $22 \%(5 / 23)$ and $38 \%(8 / 21)$, respectively. Compared with nivolumab monotherapy, nivolumab combined with ipilimumab resulted in higher PCR (10\% vs. 38\%), and less viable tumor (median $50 \%$ vs. $9 \%$ ). In a phase II clinical trial of atezolizumab combined with chemotherapy (20), after two cycles of treatment, the MPR rate increased to $57 \%$ (17/30). The NADIM trial from Spain (43), a total of 46 patients were enrolled and given nivolumab combined with chemotherapy. The results reported that among 41 patients undergoing surgery, 83\% (34/41) achieved MPR and $63 \%(26 / 41)$ achieved PCR. Furthermore, combined immunotherapy was found to increase the frequency of tumor-infiltrating lymphocytes; no unacceptable toxicity 
was found, nor was an increase in perioperative morbidity/ mortality observed. The results of these trials further increased the need to define the value MPR as a surrogate endpoint for survival in neoadjuvant therapy. Currently, a number of phase III neoadjuvant immunotherapy clinical studies are underway, including CheckMate816 (44), KEYNOTE-671 (45), IMpower030 (46), AEGEAN (47), etc. The main research endpoints include MPR.

\section{Standardization of patbological evaluation}

Chemotherapy, oncogene targeted therapy, and immunotherapy have different mechanisms of action, and different treatment-related characteristics can be observed on resected specimens post-neoadjuvant therapy. Originally, Pataer et al. (37) proposed to obtain at least 1 section per centimeter of the maximum tumor diameter, and then average the results of all slides. They reviewed a large number of histological criteria, with the main 3 characteristics including necrosis, stromal fibrosis, and live tumor. It was proposed that the percentage of live tumors is the only histological index with predictive significance. Hellmann et al. (40) modified this method, and subsequently, Cottrell et al. proposed to establish a new immunerelated pathological response standard (irPRC) (48), with the area of "return bed" being increased to the area of remaining viable tumor and necrosis. In addition, the terms "stroma", "fibrosis", and "inflammation" were described in greater detail as tissue repair-neovascularization and proliferative fibrosis, dense tumor-infiltrating lymphocytes, and tertiary lymphoid structures. It was concluded that irPRC can be used for pathological evaluation of standardized immunotherapy efficacy. However, long-term follow-up is needed to determine the reliability of irPRC as a surrogate indicator of RFS and OS. Examination of MPR score should perform by two pathologists independently. All pathologists need have some training regarding MPR scoring. There are few data on the repeatability of MPR assessments. In the study of Qu et al. (49), they used intraclass correlation (ICC) to quantify the Interobserver agreement and Bland-Altman diagram to show the level of difference. The results showed that in the assessment of adenocarcinoma (ADC) (ICC $=0.97 ; 95 \%$ CI, 0.93-0.99) and squamous cell carcinoma (SCC) (ICC $=0.99 ; 95 \%$ CI, $0.96-1.00)$ by two pathologists, there was a high degree of interobserver reproducibility. In addition, in the study of Blaauwgeers et al. (50), two experienced lung pathologists independently scored the proportion and distribution of important tumors. A 10\% cutoff for viable tumor was used to evaluate the relationship between histopathological changes and clinical outcomes. The final result found that there was no difference in the scores between the two pathologists.

The International Association for the Study of Lung Cancer (IASLC) has made detailed recommendations on how to handle lung cancer resection specimens and define pathological responses. This method can be used for all systemic therapies (51). A standardized method is recommended to assess the percentage of live tumors, necrosis, and stroma (including inflammation and fibrosis), makes these add up to $100 \%$. In addition, specific recommendations are made for the pathological evaluation of lymph nodes in patients with lung cancer. They pointed out that when assessing lymph node metastasis, the same methods as recommended for primary lung tumors can be used. And it is suggested that if there is a clear scar and, or tumor necrosis area in the absence of discernible viable tumor cells, then PCR in the lymph nodes can be recognized. However, in lymph node metastatic lung cancer, the detailed histological characteristics have not yet been determined.

\section{Other potential secondary endpoints}

\section{Metabolic response PET}

After neoadjuvant chemotherapy for NSCLC, the standardized uptake value (SUV) (max, corr) obtained by 2 consecutive PET scans can predict the histopathological response of the primary tumor and mediastinal lymph nodes, and has prognostic value (52). Studies have shown that for patients with an SUV $(\max )$ reduction in primary tumors after neoadjuvant chemotherapy of more than $60 \%$ under PET imaging, the 5 -year OS rate is significantly higher (53). Pöttgen et al. (54) analyzed PET scans before and after treatment in a neoadjuvant trial, and the results showed that in univariate and multivariate analysis, remaining maintained as a continuous variable that could predict OS, PFS, and presence of extracerebral metastasis $(\mathrm{P}<0.016)$. Furthermore, for every $50 \%$ increase in SUV\%, the HR of these 3 endpoints also increased significantly. They thus reasoned that \% SUV remaining is a predictor for survival and other end points after multimodality treatment. PET can be used in the selection and application of neoadjuvant chemotherapy. Chaft et al. (55) used 18-F fluorodeoxyglucose (18F-FDG) PET scans on 40 patients 
with stage IB-IIIA lung cancer who received 2 cycles of cisplatin chemotherapy. If the SUV in the primary tumor was reduced by at least $35 \%$, the patient continued with initial therapy. Patients with a PET response lower than $35 \%$ were switched to vinorelbine plus docetaxel. The endpoint was defined as the response rate of previously unresponsive patients to the new chemotherapy regimen. There were 15 patients whose SUV decreased by less than $35 \%$, and 13 patients received vinorelbine plus docetaxel treatment. 10 of 13 patients reached their primary endpoint (67\%). Furthermore, 6 out of 40 patients achieved MPR. Studies have shown that $18 \mathrm{~F}-\mathrm{FDG}$ PET can evaluate the patient's response to different chemotherapy regimens by measuring the decline in SUV before and after medication. This adaptive method can also be used to test new drugs in an attempt to optimize perioperative chemotherapy for better long-term efficacy.

Moreover, 18F-FDG PET can also be used to predict pathological results after neoadjuvant immunotherapy. Previously, Lopci et al. analyzed the relationship between FDG-PET metabolic information and the tissue expression of immune markers in patients with NSCLC after surgical resection (56). It was found that the maximum value of SUV and the average value of SUV were directly related to the expression of PD-1 (rho $=0.33 ; \mathrm{P}=0.017$ and rho $=0.36$; $\mathrm{P}=0.009)$. Studies have also shown that the high expression level of PD-L1 is closely related to 18 F-FDG uptake, glucose metabolism, and hypoxia. PD-L1 and SUV max are independent prognostic predictors (57). Recently, studies have evaluated the relationship between the tumor metabolism parameters of 18 F-FDG PET and the pathological response of neoadjuvant sintilimab in patients with resectable NSCLC. The results showed that in 13 of the 36 tumors removed (36.1\%), MPR was achieved; PET scans showed that 13 patients (36.1\%) had partial metabolic response (PMR). All (100.0\%) PMR ( $\triangle$ SUL peak $\%<-30.0 \%$ ) tumors showed MPR. This proves that the metabolic response caused by $18 \mathrm{~F}-\mathrm{FDG}$ is significantly related to the pathological response after neoadjuvant immunotherapy and can predict the MPR rate of resectable NSCLC after neoadjuvant therapy (58).

\section{T-cell response}

A preclinical study evaluating a triple-negative breast cancer model found that neoadjuvant immunotherapy can increase tumor-specific CD8+ T cells in peripheral blood and organs, thereby suggesting that the increase and continuous maintenance of peripheral tumor-specific immune response can enhance the antitumor efficacy induced by neoadjuvant immunotherapy. Blood sampling of tumor-specific CD8+ $\mathrm{T}$ cells immediately before and after surgery could provide a predictive indicator of results (59). In resectable NSCLC, some researchers have used tumor/blood samples from phase II clinical trials to explore whether peripheral T-cell clonal dynamics can predict the pathological response after neoadjuvant PD-1 blockade (60). The results show that the T-cell receptor (TCR) repertoire reaching MPR has a higher clonality and a greater tumor invasion rate. A higher intratumoral TCR clonotype is associated with a decrease in the percentage of residual tumors during surgery. The study suggested that exchange of T-cell clones between tumor and blood represents a key correlate of pathologic response to neoadjuvant immunotherapy and can be used to predict the pathological results of NSCLC patients.

\section{ctDNA analysis}

Deep sequencing (CAPP-seq) ctDNA analysis may represent a promising approach for monitoring responses during and after neoadjuvant therapy. Research has suggested that residual ctDNA after curative-intent surgery or radiotherapy of localized lung cancer is a sensitive and specific marker of patients at the highest risk of recurrence. Chaudhuri et al. (61) conducted analysis on 255 samples from 40 cured lung cancer patients and 54 healthy adults. The results showed that $53 \%$ of the patients had ctDNA mutation characteristics, which were related to blockade of tyrosine kinase inhibitors or immune checkpoints. This indicates that ctDNA analysis during adjuvant chemotherapy can strongly identify posttreatment molecular residual disease (MRD) in patients with localized lung cancer. Future trials should test its ability to predict treatment response early in neoadjuvant therapy, its correlation with MPR and pCR, and its ability to identify patients who may receive other treatments after surgery.

\section{Summary and prospect}

The main goals of neoadjuvant trials are to increase chances of cure, more quickly identify drugs that can improve prognosis after complete resection, and to standardize endpoints and trial design, so that neoadjuvant results can be used to accelerate drug development and approval. Traditional research endpoints in the curative setting such as OS, DFS, etc. consume considerable resources and time. In order to accelerate the development and approval 
of neoadjuvant drugs, new biomarkers that can be used as surrogate endpoints should be sought to record the impact on long-term survival.

The pathological response after surgical resection is an important factor in evaluating the efficacy of neoadjuvant therapy. Nowadays, there is an abundance of data that can be used to analyze whether pathological response criteria, such as MPR or pCR, are viable as surrogate endpoints. A significant correlation between MPR or pCR and EFS and OS has been demonstrated. With the development of ICIs, the pathological response to EFS or OS may be different from the pathological response to chemotherapy or targeted therapy. Therefore, the definitions of MPR and pCR should be standardized, and a new standard should be developed that can be used in all ongoing and planned neoadjuvant immunotherapy trials, neoadjuvant targeted therapy trials, and combination therapy. The residual percentage of surviving tumor cells in resected NSCLC patients is also a valuable measure. It can provide more accurate and rapid comparisons between different neoadjuvant treatment options, thereby reducing the time required to evaluate new drugs in clinical trials.

However, there are still many issues to be resolved, such as the balance and unification between imaging evaluation and histopathological evaluation, the selection of other biomarkers for accurate prediction of MPR or pCR, and the need for more prospective trials and meta-analyses to determine if MPR and pCR can predict survival uniformly across treatment modalities or whether different rules will need to be applied for different kinds of treatment. In addition, further study of the association between MPR and longer-term outcomes in various histologies, genetic subgroups, and pharmacologic classes of neoadjuvant therapies should be explored and more suitable clinical alternative endpoints should be sought.

\section{Acknowledgments}

The authors appreciate the academic support from the AME Lung Cancer Collaborative Group.

Funding: This study was supported by the National Nature Science Foundation in China (NSFC) (grant number: 82002400) and the Natural Science Foundation of Zhejiang Provincial (grant number: Y19H160116, Q18H160119).

\section{Footnote}

Reporting Checklist: The authors have completed the
Narrative Review reporting checklist. Available at http:// dx.doi.org/10.21037/tlcr-21-259

Conflicts of Interest: All authors have completed the ICMJE uniform disclosure form (available at http://dx.doi. org/10.21037/tlcr-21-259). Dr. Guibert reports personal fees from Astra Zeneca, Roche, BMS, MSD, AMGEN, Novartis, Pfizer, Chiesi, outside the submitted work. Dr. $\mathrm{RC}$ has received honoraria for lectures, presentations, speakers bureaus, educational events and advisory boards from Astrazeneca, Roche, BMS and MSD, and support for attending meetings and/or travel from MSD and Roche, outside the submitted work. The other authors have no conflicts of interest to declare.

Ethical Statement: The authors are accountable for all aspects of the work in ensuring that questions related to the accuracy or integrity of any part of the work are appropriately investigated and resolved.

Open Access Statement: This is an Open Access article distributed in accordance with the Creative Commons Attribution-NonCommercial-NoDerivs 4.0 International License (CC BY-NC-ND 4.0), which permits the noncommercial replication and distribution of the article with the strict proviso that no changes or edits are made and the original work is properly cited (including links to both the formal publication through the relevant DOI and the license). See: https://creativecommons.org/licenses/by-nc-nd/4.0/.

\section{References}

1. Asamura H, Chansky K, Crowley J, et al. The International Association for the Study of Lung Cancer Lung Cancer Staging Project: Proposals for the Revision of the N Descriptors in the Forthcoming 8th Edition of the TNM Classification for Lung Cancer. J Thorac Oncol 2015;10:1675-84.

2. Blumenthal GM, Bunn PA Jr, Chaft JE, et al. Current Status and Future Perspectives on Neoadjuvant Therapy in Lung Cancer. J Thorac Oncol 2018;13:1818-31.

3. Eberhardt WE, Mitchell A, Crowley J, et al. The IASLC Lung Cancer Staging Project: Proposals for the Revision of the M Descriptors in the Forthcoming Eighth Edition of the TNM Classification of Lung Cancer. J Thorac Oncol 2015;10:1515-22.

4. Rami-Porta R, Bolejack V, Crowley J, et al. The IASLC Lung Cancer Staging Project: Proposals for the Revisions 
of the T Descriptors in the Forthcoming Eighth Edition of the TNM Classification for Lung Cancer. J Thorac Oncol 2015;10:990-1003.

5. NSCLC Meta-analysis Collaborative Group. Preoperative chemotherapy for non-small-cell lung cancer: a systematic review and meta-analysis of individual participant data. Lancet 2014;383:1561-71.

6. NSCLC Meta-analyses Collaborative Group; Arriagada R, Auperin A, et al. Adjuvant chemotherapy, with or without postoperative radiotherapy, in operable non-small-cell lung cancer: two meta-analyses of individual patient data. Lancet 2010;375:1267-77.

7. Postmus PE, Kerr KM, Oudkerk M, et al. Early and locally advanced non-small-cell lung cancer (NSCLC): ESMO Clinical Practice Guidelines for diagnosis, treatment and follow-up. Ann Oncol 2017;28:iv1-iv21.

8. Pless M, Stupp R, Ris HB, et al. Induction chemoradiation in stage IIIA/N2 non-small-cell lung cancer: a phase 3 randomised trial. Lancet 2015;386:1049-56.

9. Xu YP, Li B, Xu XL, et al. Is There a Survival Benefit in Patients With Stage IIIA (N2) Non-small Cell Lung Cancer Receiving Neoadjuvant Chemotherapy and/or Radiotherapy Prior to Surgical Resection: A Systematic Review and Meta-analysis. Medicine (Baltimore) 2015;94:e879.

10. Rusch VW, Giroux DJ, Kraut MJ, et al. Induction chemoradiation and surgical resection for superior sulcus non-small-cell lung carcinomas: long-term results of Southwest Oncology Group Trial 9416 (Intergroup Trial 0160). J Clin Oncol 2007;25:313-8.

11. Nagano T, Tachihara M, Nishimura Y. Molecular Mechanisms and Targeted Therapies Including Immunotherapy for Non-Small Cell Lung Cancer. Curr Cancer Drug Targets 2019;19:595-630.

12. Zhong WZ, Wang Q, Mao WM, et al. Gefitinib versus vinorelbine plus cisplatin as adjuvant treatment for stage II-IIIA (N1-N2) EGFR-mutant NSCLC (ADJUVANT/ CTONG1104): a randomised, open-label, phase 3 study. Lancet Oncol 2018;19:139-48.

13. Zhong WZ, Chen KN, Chen C, et al. Erlotinib Versus Gemcitabine Plus Cisplatin as Neoadjuvant Treatment of Stage IIIA-N2 EGFR-Mutant Non-Small-Cell Lung Cancer (EMERGING-CTONG 1103): A Randomized Phase II Study. J Clin Oncol 2019;37:2235-45.

14. Paz-Ares L, Luft A, Vicente D, et al. Pembrolizumab plus Chemotherapy for Squamous Non-Small-Cell Lung Cancer. N Engl J Med 2018;379:2040-51.

15. Hellmann MD, Ciuleanu TE, Pluzanski A, et al.
Nivolumab plus Ipilimumab in Lung Cancer with a High Tumor Mutational Burden. N Engl J Med 2018;378:2093-104.

16. Socinski MA, Jotte RM, Cappuzzo F, et al. Atezolizumab for First-Line Treatment of Metastatic Nonsquamous NSCLC. N Engl J Med 2018;378:2288-301.

17. Forde PM, Chaft JE, Smith KN, et al. Neoadjuvant PD-1 Blockade in Resectable Lung Cancer. N Engl J Med 2018;378:1976-86.

18. Kwiatkowski DJ, Rusch VW, Chaft JE, et al. Neoadjuvant atezolizumab in resectable non-small cell lung cancer (NSCLC): interim analysis and biomarker data from a multicenter study (LCMC3). J Clin Oncol 2019;37:abstr 8503.

19. Yotsukura M, Nakagawa K, Suzuki K, et al. Recent advances and future perspectives in adjuvant and neoadjuvant immunotherapies for lung cancer. Jpn J Clin Oncol 2021;51:28-36.

20. Shu CA, Gainor JF, Awad MM, et al. Neoadjuvant atezolizumab and chemotherapy in patients with resectable non-small-cell lung cancer: an open-label, multicentre, single-arm, phase 2 trial. Lancet Oncol 2020;21:786-95.

21. Jia XH, Xu H, Geng LY, et al. Efficacy and safety of neoadjuvant immunotherapy in resectable nonsmall cell lung cancer: A meta-analysis. Lung Cancer 2020;147:143-53.

22. Sepesi B, Godoy M, William W, et al. P2.04-90 Nodal Immune Flare (NIF) Following Neoadjuvant Anti-PD-1 and Anti-CTLA-4 Therapy in Non-Small Cell Lung Cancer. J Thorac Oncol 2019;14:S745.

23. Kilickap S, Demirci U, Karadurmus N, et al. Endpoints in oncology clinical trials. J BUON 2018;23:1-6.

24. Fiteni F, Westeel V, Pivot X, et al. Endpoints in cancer clinical trials. J Visc Surg 2014;151:17-22.

25. Sacher AG, Le LW, Leighl NB. Shifting patterns in the interpretation of phase III clinical trial outcomes in advanced non-small-cell lung cancer: the bar is dropping. J Clin Oncol 2014;32:1407-11.

26. Fiteni F, Westeel V, Bonnetain F. Surrogate endpoints for overall survival in lung cancer trials: a review. Expert Rev Anticancer Ther 2017;17:447-54.

27. Mauguen A, Pignon JP, Burdett S, et al. Surrogate endpoints for overall survival in chemotherapy and radiotherapy trials in operable and locally advanced lung cancer: a re-analysis of meta-analyses of individual patients' data. Lancet Oncol 2013;14:619-26.

28. Montemurro F, Di Cosimo S. Pathological complete response in breast cancer patients receiving neoadjuvant 
chemotherapy. Breast 2014;23:690-1.

29. Cortazar P, Zhang L, Untch M, et al. Pathological complete response and long-term clinical benefit in breast cancer: the CTNeoBC pooled analysis. Lancet 2014;384:164-72.

30. Schmid P, Cortes J, Pusztai L, et al. Pembrolizumab for Early Triple-Negative Breast Cancer. N Engl J Med 2020;382:810-21.

31. Pisters KM, Kris MG, Gralla RJ, et al. Pathologic complete response in advanced non-small-cell lung cancer following preoperative chemotherapy: implications for the design of future non-small-cell lung cancer combined modality trials. J Clin Oncol 1993;11:1757-62.

32. Depierre A, Milleron B, Moro-Sibilot D, et al. Preoperative chemotherapy followed by surgery compared with primary surgery in resectable stage I (except T1N0), II, and IIIa non-small-cell lung cancer. J Clin Oncol 2002;20:247-53.

33. Betticher DC, Hsu Schmitz SF, Tötsch M, et al. Mediastinal lymph node clearance after docetaxel-cisplatin neoadjuvant chemotherapy is prognostic of survival in patients with stage IIIA pN2 non-small-cell lung cancer: a multicenter phase II trial. J Clin Oncol 2003;21:1752-9.

34. Betticher DC, Hsu Schmitz SF, Tötsch M, et al. Prognostic factors affecting long-term outcomes in patients with resected stage IIIA pN2 non-small-cell lung cancer: 5-year follow-up of a phase II study. Br J Cancer 2006;94:1099-106.

35. Mouillet G, Monnet E, Milleron B, et al. Pathologic complete response to preoperative chemotherapy predicts cure in early-stage non-small-cell lung cancer: combined analysis of two IFCT randomized trials. J Thorac Oncol 2012;7:841-9.

36. Junker K, Langner K, Klinke F, et al. Grading of tumor regression in non-small cell lung cancer : morphology and prognosis. Chest 2001;120:1584-91.

37. Pataer A, Kalhor N, Correa AM, et al. Histopathologic response criteria predict survival of patients with resected lung cancer after neoadjuvant chemotherapy. J Thorac Oncol 2012;7:825-32.

38. William WN Jr, Pataer A, Kalhor N, et al. Computed tomography RECIST assessment of histopathologic response and prediction of survival in patients with resectable non-small-cell lung cancer after neoadjuvant chemotherapy. J Thorac Oncol 2013;8:222-8.

39. Chaft JE, Rusch V, Ginsberg MS, et al. Phase II trial of neoadjuvant bevacizumab plus chemotherapy and adjuvant bevacizumab in patients with resectable nonsquamous non-small-cell lung cancers. J Thorac Oncol 2013;8:1084-90.

40. Hellmann MD, Chaft JE, William WN Jr, et al. Pathological response after neoadjuvant chemotherapy in resectable non-small-cell lung cancers: proposal for the use of major pathological response as a surrogate endpoint. Lancet Oncol 2014;15:e42-50.

41. Bott MJ, Yang SC, Park BJ, et al. Initial results of pulmonary resection after neoadjuvant nivolumab in patients with resectable non-small cell lung cancer. J Thorac Cardiovasc Surg 2019;158:269-76.

42. Cascone T, William WN Jr, Weissferdt A, et al. Neoadjuvant nivolumab or nivolumab plus ipilimumab in operable non-small cell lung cancer: the phase 2 randomized NEOSTAR trial. Nat Med 2021;27:504-14.

43. Provencio M, Nadal E, Insa A, et al. Neoadjuvant chemotherapy and nivolumab in resectable non-small-cell lung cancer (NADIM): an open-label, multicentre, singlearm, phase 2 trial. Lancet Oncol 2020;21:1413-22.

44. ClinicalTrials.gov. A neoadjuvant study of nivolumab plus ipilimumab plus chemotherapy versus chemotherapy alone in early stage non-small cell lung cancer (NSCLC) (CheckMate816). Available online: https://clinicaltrials. gov/ct2/show/NCT02998528

45. ClinicalTrials.gov. Efficacy and safety of pembrolizumab (MK-3475) with platinum doublet chemotherapy as neoadjuvant/adjuvant therapy for participants with resectable stage IIB or IIIA non-small cell lung cancer (MK-3475-671/KEYNOTE-671). Available online: https://clinicaltrials.gov/ct2/show/NCT03425643

46. ClinicalTrials.gov. A Study of Neoadjuvant Atezolizumab Plus Chemotherapy Versus Placebo Plus Chemotherapy in Patients With Resectable Stage II, IIIA, or Select IIIB Non-Small Cell Lung Cancer (IMpower030). Available online: https://clinicaltrials. gov/ct2/show/NCT03456063

47. ClinicalTrials.gov. A Study of Neoadjuvant/Adjuvant Durvalumab for the Treatment of Patients With Resectable Non-small Cell Lung Cancer. Available online: https://clinicaltrials.gov/ct2/show/NCT03800134

48. Cottrell TR, Thompson ED, Forde PM, et al. Pathologic features of response to neoadjuvant anti-PD-1 in resected non-small-cell lung carcinoma: a proposal for quantitative immune-related pathologic response criteria (irPRC). Ann Oncol 2018;29:1853-60.

49. Qu Y, Emoto K, Eguchi T, et al. Pathologic Assessment After Neoadjuvant Chemotherapy for NSCLC: Importance and Implications of Distinguishing 
Adenocarcinoma From Squamous Cell Carcinoma. J Thorac Oncol 2019;14:482-93.

50. Blaauwgeers JL, Kappers I, Klomp HM, et al. Complete pathological response is predictive for clinical outcome after tri-modality therapy for carcinomas of the superior pulmonary sulcus. Virchows Arch 2013;462:547-56.

51. Travis WD, Dacic S, Wistuba I, et al. IASLC Multidisciplinary Recommendations for Pathologic Assessment of Lung Cancer Resection Specimens After Neoadjuvant Therapy. J Thorac Oncol 2020;15:709-40.

52. Pöttgen C, Levegrün S, Theegarten D, et al. Value of 18F-fluoro-2-deoxy-D-glucose-positron emission tomography/computed tomography in non-small-cell lung cancer for prediction of pathologic response and times to relapse after neoadjuvant chemoradiotherapy. Clin Cancer Res 2006;12:97-106.

53. Dooms C, Verbeken E, Stroobants S, et al. Prognostic stratification of stage IIIA-N2 non-small-cell lung cancer after induction chemotherapy: a model based on the combination of morphometric-pathologic response in mediastinal nodes and primary tumor response on serial 18-fluoro-2-deoxy-glucose positron emission tomography. J Clin Oncol 2008;26:1128-34.

54. Pöttgen C, Gauler T, Bellendorf A, et al. Standardized Uptake Decrease on 18F-Fluorodeoxyglucose Positron Emission Tomography After Neoadjuvant Chemotherapy Is a Prognostic Classifier for Long-Term Outcome After Multimodality Treatment: Secondary Analysis of a Randomized Trial for Resectable Stage IIIA/B Non-SmallCell Lung Cancer. J Clin Oncol 2016;34:2526-33.

55. Chaft JE, Dunphy M, Naidoo J, et al. Adaptive

Cite this article as: Ren S, Xu A, Lin Y, Camidge DR, Di Maio M, Califano R, Hida T, Rossi A, Guibert N, Zhu C, Shen J. A narrative review of primary research endpoints of neoadjuvant therapy for lung cancer: past, present and future. Transl Lung Cancer Res 2021;10(7):3264-3275. doi: 10.21037/tlcr-21-259
Neoadjuvant Chemotherapy Guided by (18)F-FDG PET in Resectable Non-Small Cell Lung Cancers: The NEOSCAN Trial. J Thorac Oncol 2016;11:537-44.

56. Lopci E, Toschi L, Grizzi F, et al. Correlation of metabolic information on FDG-PET with tissue expression of immune markers in patients with non-small cell lung cancer (NSCLC) who are candidates for upfront surgery. Eur J Nucl Med Mol Imaging 2016;43:1954-61.

57. Kaira K, Shimizu K, Kitahara S, et al. 2-Deoxy-2fluorine-18 fluoro-d-glucose uptake on positron emission tomography is associated with programmed death ligand-1 expression in patients with pulmonary adenocarcinoma. Eur J Cancer 2018;101:181-90.

58. Tao X, Li N, Wu N, et al. The efficiency of 18F-FDG PET-CT for predicting the major pathologic response to the neoadjuvant PD-1 blockade in resectable nonsmall cell lung cancer. Eur J Nucl Med Mol Imaging 2020;47:1209-19.

59. Liu J, Blake SJ, Yong MC, et al. Improved Efficacy of Neoadjuvant Compared to Adjuvant Immunotherapy to Eradicate Metastatic Disease. Cancer Discov 2016;6:1382-99.

60. Zhang J, Ji Z, Caushi JX, et al. Compartmental Analysis of T-cell Clonal Dynamics as a Function of Pathologic Response to Neoadjuvant PD-1 Blockade in Resectable Non-Small Cell Lung Cancer. Clin Cancer Res 2020;26:1327-37.

61. Chaudhuri AA, Chabon JJ, Lovejoy AF, et al. Early Detection of Molecular Residual Disease in Localized Lung Cancer by Circulating Tumor DNA Profiling. Cancer Discov 2017;7:1394-403. 